\title{
Recoilless resonant neutrino experiment and origin of neutrino oscillations 1
}

\author{
S. M. Bilenky \\ Joint Institute for Nuclear Research, Dubna, R-141980, Russia \\ F. von Feilitzsch and W. Potzel \\ Physik-Department E15, Technische Universität München, D-85748 \\ Garching, Germany
}

\begin{abstract}
We demonstrate that an experiment with recoilless resonant emission and absorption of tritium $\bar{\nu}_{e}$ could have an important impact on our understanding of the origin of neutrino oscillations.
\end{abstract}

\section{Introduction}

Evidence for neutrino oscillations obtained in the Super-Kamiokande atmospheric [1], SNO solar [2], KamLAND reactor [3] and other neutrino experiments [4, 5, 6, 7, 8, 9] is one of the most important recent discoveries in particle physics. Small neutrino masses can not be generatad by the Standard Higgs mechanism. Some new mechanism of mass generation is necessary.

All existing neutrino oscillation data can be explained by three-neutrino mixing 2 In the framework of three-neutrino mixing, from the analysis of the Super-Kamiokande atmospheric neutrino data the following ranges for the largest neutrino mass-squared difference $\Delta m_{23}^{2}$ and for the mixing angle were obtained $[1]^{3}$

$$
1.5 \cdot 10^{-3} \leq \Delta m_{23}^{2} \leq 3.4 \cdot 10^{-3} \mathrm{eV}^{2} ; \quad \sin ^{2} 2 \theta_{23}>0.92 .
$$

From a global analysis of the KamLAND and solar neutrino data it was found 3

$$
\Delta m_{12}^{2}=7.9_{-0.5}^{+0.6} 10^{-5} \mathrm{eV}^{2} ; \quad \tan ^{2} \theta_{12}=0.40_{-0.07}^{+0.10}
$$

\footnotetext{
${ }^{1}$ The report at the Workshop on Next Generation Nucleon Decay and Neutrino Detectors, NNN06, September 21-23, 2006, University of Washington, Seattle, USA.

${ }^{2}$ Recent results of the MiniBooNE experiment 10] do not confirm the LSND indication 11 in favor of existence of more than three massive neutrinos.

${ }^{3}$ Neutrino mass-squared difference is determined as follows: $\Delta m_{i k}^{2}=m_{k}^{2}-m_{i}^{2}$.
} 
Only an upper bound for the angle $\theta_{13}$ is known at present [12]

$$
\sin ^{2} \theta_{13} \leq 5 \cdot 10^{-2} .
$$

In future neutrino oscillation experiments the accuracies of the neutrino oscillation parameters are planned to be improved. One of the major aims of future experiments is to determine the value of the parameter $\theta_{13}$ which is crucial for the measurement of the $C P$ violation in the lepton sector and the determination of the character of the neutrino mass spectrum.

From the point of view of field theory, the phenomenon of neutrino oscillations is based on the following assumptions:

1. Neutrino interactions are the SM charged current (CC) and neutral current $(\mathrm{NC})$ interactions. The leptonic $\mathrm{CC}$ and neutrino $\mathrm{NC}$ are given by

$$
j_{\alpha}^{\mathrm{CC}}(x)=2 \sum_{l=e, \mu, \tau} \bar{\nu}_{l L}(x) \gamma_{\alpha} l_{L}(x) ; \quad j_{\alpha}^{\mathrm{NC}}(x)=\sum_{l=e, \mu, \tau} \bar{\nu}_{l L}(x) \gamma_{\alpha} \nu_{l L}(x) .
$$

2. The fields of neutrinos with definite masses enter into CC and $\mathrm{NC}$ in the mixed form

$$
\nu_{l L}(x)=\sum_{k=1}^{3} U_{l k} \nu_{k L}(x) .
$$

Here $\nu_{k}(x)$ is the field of neutrino with mass $m_{k}$ and $U$ is the unitary PMNS matrix [13, 14].

Due to neutrino mixing, Eq.(5), the flavor lepton numbers $L_{e}, L_{\mu}$ and $L_{\tau}$ are not conserved in neutrino transitions. The standard probability of the transition $\nu_{l} \rightarrow \nu_{l^{\prime}}$ is given by (see [15])

$$
P\left(\nu_{l} \rightarrow \nu_{l^{\prime}}\right)=\left|\sum_{k=1}^{3} U_{l^{\prime} k} e^{-i \Delta m_{1 k}^{2} \frac{L}{2 E}} U_{l k}^{*}\right|^{2},
$$

where $L$ is the distance between the neutrino-detection and neutrino-production points and $E$ is the neutrino energy. Expression (6) perfectly describes the existing neutrino oscillation data. Let us notice that taking into account the unitarity of the mixing matrix we can rewrite (6) in a form

$$
P\left(\nu_{l} \rightarrow \nu_{l^{\prime}}\right)=\left|\delta_{l^{\prime} l}+\sum_{k \neq 1} U_{l^{\prime} k}\left(e^{-i \Delta m_{1 k}^{2} \frac{L}{2 E}}-1\right) U_{l k}^{*}\right|^{2} .
$$


The probabilities $P\left(\nu_{l} \rightarrow \nu_{l^{\prime}}\right)$ depend on six parameters. However, $\frac{\Delta m_{12}^{2}}{\Delta m_{23}^{2}} \ll 1$ and $\sin ^{2} \theta_{13} \ll 1$. In first approximation we can neglect the contributions of these parameters to the transition probabilities. In this approximation neutrino oscillations in the atmospheric-LBL and solar-KamLAND regions are described by the simplest two-neutrino expressions which depend, correspondingly, on $\Delta m_{23}^{2}, \sin ^{2} 2 \theta_{23}$ and $\Delta m_{12}^{2}, \tan ^{2} \theta_{12}$ (see review [15]). The numerical values of these parameters given in (11) and (2) were obtained from the analysis of the experimental data by using two-neutrino expressions.

Several derivations of Eq. (6), based on different physical assumptions, exist in the literature. It is not possible to test these assumptions in usual neutrino oscillation experiments. We will show here that an experiment on resonant recoilless emission and capture of tritium $\bar{\nu}_{e}$ 's, proposed recently in [16, 17], could provide such a possibility (for additional information, see [18]).

\section{Different approaches to neutrino oscillations}

We discuss here different points of view on the origin of neutrino oscillations. Neutrinos are produced in $\mathrm{CC}$ weak processes. For the difference of momenta of neutrinos with masses $m_{k}$ and $m_{i}$ (in the rest-frame of the source) we have

$$
\Delta p_{i k}=\left(p_{k}-p_{i}\right) \sim \frac{\Delta m_{i k}^{2}}{E},
$$

where $E$ is the neutrino energy. In standard neutrino oscillation experiments $E \gtrsim \mathrm{MeV}$. From (8), (11), and (2) follows that $\left|\Delta p_{i k}\right|$ is much smaller than the quantum-mechanical uncertainty of the momentum. This is the reason why in CC neutrino-production processes together with $e^{+}, \mu^{+}, \tau^{+}$, correspondingly, flavor neutrinos $\nu_{e}, \nu_{\mu}, \nu_{\tau}$, which are described by mixed flavor neutrino states,

$$
\left|\nu_{l}\right\rangle=\sum_{k=1}^{3} U_{l k}^{*}\left|\nu_{k}\right\rangle, \quad l=e, \mu, \tau
$$

are produced (see, for example, [19]). Here $\left|\nu_{k}\right\rangle$ is the state of a neutrino with mass $m_{k}$ and 4 -momentum $p_{k}=\left(E_{k}, \overrightarrow{p_{k}}\right)$.

The main difference between different approaches to neutrino oscillations is connected with assumptions about the propagation of flavor neutrino states. We will discuss two different assumptions. 


\section{Evolution in time.}

The evolution equation of any quantum system is the Schrödinger equation (see, for example, [20])

$$
i \frac{\partial|\Psi(t)\rangle}{\partial t}=H|\Psi(t)\rangle
$$

Here $|\Psi(t)\rangle$ is the state of the system at the time $t$ and $H$ is the total Hamiltonian. The general solution of this equation has the form

$$
|\Psi(t)\rangle=e^{-i H t}|\Psi(0)\rangle
$$

where $|\Psi(0)\rangle$ is the state of the system at the initial time $t=0$.

If at $t=0$ the flavor neutrino $\nu_{l}$ is produced, we have $|\Psi(0)\rangle=\left|\nu_{l}\right\rangle$ and the neutrino state in vacuum at the time $t \geq 0$ is given by

$$
\left|\nu_{l}\right\rangle_{t}=e^{-i H t}\left|\nu_{l}\right\rangle=\sum_{k=1}^{3} e^{-i E_{k} t} U_{l k}^{*}\left|\nu_{k}\right\rangle .
$$

Thus, if the energies $E_{k}$ are different, the neutrino state $\left|\nu_{l}\right\rangle_{t}$ is a nonstationary one. For such states the time-energy uncertainty relation

$$
\Delta E \Delta t \gtrsim 1
$$

holds (see, for example, [21]). In this relation, $\Delta E$ is the energy uncertainty and $\Delta t$ is the time interval during which the state of the system is significantly changed.

Neutrinos are detected via the observation of $\mathrm{CC}$ and $\mathrm{NC}$ reactions. In such reactions, flavor neutrinos $\nu_{l^{\prime}}$, which are described by mixed coherent states (91), are detected. From (9) and (12) we find

$$
\left|\nu_{l}\right\rangle_{t}=\sum_{l^{\prime}}\left|\nu_{l^{\prime}}\right\rangle \sum_{k=1}^{3} U_{l^{\prime} k} e^{-i E_{k} t} U_{l k}^{*} .
$$

Thus, the transition probability $\nu_{l} \rightarrow \nu_{l^{\prime}}$ is given by

$$
P\left(\nu_{l} \rightarrow \nu_{l^{\prime}}\right)=\left|\sum_{k=1}^{3} U_{l^{\prime} k} e^{-i\left(E_{k}-E_{1}\right) t} U_{l k}^{*}\right|^{2}
$$


From this expression it is obvious that if the energies of the neutrinos with different masses are equal, $P\left(\nu_{l} \rightarrow \nu_{l^{\prime}}\right)=\delta_{l^{\prime} l}$. Thus, in the approach based on the Schrödinger evolution equation, there will be no neutrino oscillations if $E_{k}=E_{1}[22]$.

Let us assume now that the flavor neutrino states $\left|\nu_{l}\right\rangle$ are superpositions of the neutrino states $\nu_{k}$ with the same momentum $\vec{p}$ and different energies. In the case of ultrarelativistic neutrinos, which we are interested in, we have

$$
E_{k}=\sqrt{p^{2}+m_{k}^{2}} \simeq p+\frac{m_{k}^{2}}{2 E}
$$

with $E$ being the neutrino energy at $m_{k} \rightarrow 0$.

Taking into account that

$$
t \simeq L
$$

we obtain from (15) the standard expression (6) for the transition probability. Let us note that the time-energy uncertainty relation (13) takes the form of the well-known condition for the observation of neutrino oscillations (see [23]):

$$
\left(E_{k}-E_{1}\right) t \simeq \frac{\Delta m_{1 k}^{2}}{2 E} L \gtrsim 1 .
$$

It is evident that in the approach based on the Schrödinger equation, oscillations between different flavor neutrinos are due to the fact that the neutrino state $\left|\nu_{l}\right\rangle_{t}$ is a superposition of states with different energies 4

\section{Propagation in space and time}

It has been suggested in several papers (see [24, 25, 26]) that the mixed neutrino state at the space-time point $x=(t, \vec{x})$ is given by

$$
\left|\nu_{l}\right\rangle_{x}=\sum_{k=1}^{3} e^{-i p_{k} x} U_{l k}^{*}\left|\nu_{k}\right\rangle .
$$

\footnotetext{
${ }^{4}$ We assumed that the states of flavor neutrinos are superpositions of states of neutrinos with different masses and the same momentum. Let us notice that if we assume that neutrinos with different masses have different momenta, in the expression for the transition probability in addition to the standard phases $\frac{\Delta m_{1 k}^{2}}{2 E} L$ we will have terms $\left(p_{k}-p_{1}\right) L$. These additional terms could be of the same order as the standard phases and could be different in different experiments. All analyses of neutrino oscillation data do not favor such a possibility.
} 
Here $\left|\nu_{k}\right\rangle$ is the state of a neutrino with mass $m_{k}$ and momentum $p_{k}$. From (19) we find

$$
\left|\nu_{l}\right\rangle_{x}=e^{-i p_{1} x} \sum_{l^{\prime}}\left|\nu_{l^{\prime}}\right\rangle \sum_{k=1}^{3} U_{l^{\prime} k} e^{-i\left(p_{k}-p_{1}\right) x} U_{l k}^{*}
$$

Thus,

$$
P\left(\nu_{l} \rightarrow \nu_{l^{\prime}}\right)=\left|\sum_{k=1}^{3} U_{l^{\prime} k} e^{-i\left(p_{k}-p_{1}\right) x} U_{l k}^{*}\right|^{2}
$$

is the probability to find the flavor neutrino $\nu_{l^{\prime}}$ at the point $x$ in the case that at point $x=0$ the mixed flavor neutrino $\nu_{l}$ was produced. For the phase difference we have

$$
\left(p_{k}-p_{1}\right) x=\left(E_{k}-E_{1}\right) t-\left(\left|\overrightarrow{p_{k}}\right|-\left|\overrightarrow{p_{1}}\right|\right) L=\frac{E_{k}^{2}-E_{1}^{2}}{E_{k}+E_{1}} t-\left(\left|\overrightarrow{p_{k}}\right|-\left|\overrightarrow{p_{1}}\right|\right) L
$$

where $\overrightarrow{p_{k}}=\left|\overrightarrow{p_{k}}\right| \vec{k}$ and $\vec{k} \vec{x}=L, \vec{k}$ being the unit vector in the direction of the neutrino momenta.

In the framework of the propagation of the flavor states in time and space two scenarios were considered in the literature.

\section{Scenario I. $p_{k} \neq p_{1}, t \simeq L$.}

From (22) we find for the oscillation-phase difference

$$
\left(p_{k}-p_{1}\right) x=\frac{E_{k}^{2}-E_{1}^{2}}{E_{k}+E_{1}} t-\left(\left|\overrightarrow{p_{k}}\right|-\left|\overrightarrow{p_{1}}\right|\right) L \simeq\left(\left|\overrightarrow{p_{k}}\right|-\left|\overrightarrow{p_{1}}\right|\right)\left(\frac{\left|\overrightarrow{p_{k}}\right|+\left|\overrightarrow{p_{1}}\right|}{E_{k}+E_{1}} t-L\right)+\frac{\Delta m_{1 k}^{2}}{2 E} t
$$

If we assume that the distance and the time are connected by the relation $t \simeq L$, we can neglect the first term in (23) and come to the standard oscillation-phase difference

$$
\left(p_{k}-p_{1}\right) x \simeq \frac{\Delta m_{1 k}^{2}}{2 E} L
$$

and to the standard expression (66) for the transition probability. 
Scenario II. $\quad E_{k}=E_{1}$, stationary states.

It has been suggested in [27, 24, 25] that time is not measured in neutrino oscillation experiments and in order to oscillate neutrinos with different masses must have the same energies. In other words it has been suggested in [27, 24, 25] that the flavor neutrino states are stationary ones. Taking into

account that in this case $\left|\overrightarrow{p_{k}}\right|=E-\frac{m_{k}^{2}}{2 E}$, from (22) we obtain the standard expression for the oscillation-phase difference

$$
\left(p_{k}-p_{1}\right) x=\frac{\Delta m_{1 k}^{2}}{2 E} L
$$

and the standard expression (6) for the probability of the transition between different flavor neutrinos.

Thus, in all three cases which we have considered we came to the same expression (6) for the transition probability. This means that in usual neutrino oscillation experiments it is impossible to distinguish these three cases.

Recently, a new type of neutrino experiment, based on the Mössbauer effect, has been proposed [16, 17]. In the next section we will discuss this proposal from the point of view of providing a possibility to distinguish the different assumptions on the propagation of mixed neutrino states.

\section{Recoilless resonant emission and absorp- tion of tritium $\bar{\nu}_{e}$ 's}

In [16], an experiment has been proposed for the detection of $\bar{\nu}_{e}$ with energy $\simeq 18.6 \mathrm{keV}$ in recoilless resonant (Mössbauer) transitions:

$$
{ }^{3} \mathrm{H} \rightarrow{ }^{3} \mathrm{He}+\bar{\nu}_{\mathrm{e}} ; \quad \bar{\nu}_{\mathrm{e}}+{ }^{3} \mathrm{He} \rightarrow{ }^{3} \mathrm{H} .
$$

It was estimated in [16] that the cross section of the resonant absorption of $\bar{\nu}_{e}$ by ${ }^{3} \mathrm{He}$ is equal to $\sigma_{R} \simeq 3 \cdot 10^{-33} \mathrm{~cm}^{2}$.

The study of neutrino oscillations, driven by $\Delta m_{23}^{2}$, in an experiment with neutrinos produced and detected according to (26) was proposed in [16]. In such an experiment with a baseline of $\sim 10 \mathrm{~m}$ the parameter $\sin ^{2} \theta_{13}$ can be measured.

It was estimated in [16] that the uncertainty in energy of the produced antineutrinos in the proposed experiment is of the order

$$
\Delta E \simeq 8.6 \cdot 10^{-12} \mathrm{eV} .
$$


This value of $\Delta E$ is much smaller than the energy difference of $\nu_{3}$ and $\nu_{2}$

$$
\left(E_{3}-E_{2}\right) \sim \frac{\Delta m_{23}^{2}}{2 E} \simeq 6.5 \cdot 10^{-8} \mathrm{eV}
$$

which drive neutrino oscillations in the approach based on evolution in time.

Thus, the state of flavor $\bar{\nu}_{e}$ produced and detected in the reactions (26) is the superposition of states of neutrinos with the same energy and (because of the dispersion relation) different momenta. This means that in the experiment proposed in [16] neutrino oscillations will not be observed if the approach based on the Schrödinger evolution equation is correct. On the other hand, neutrino oscillations can be observed in this experiment if one of the Scenarios I or II, based on the propagation in space and time, is correct. Thus, an experiment with recoilless resonant emission and absorption of tritium $\bar{\nu}_{e}$ 's could have an important impact on our understanding of the origin of neutrino oscillations.

\section{Conclusion}

In conclusion we make the following remarks:

1. In an experiment proposed in [16] a positive effect of neutrino oscillations can be observed only in the case that the parameter $\sin ^{2} \theta_{13}$ is not too small. At present, only an upper bound of this parameter is known [12]. A positive result of the experiment would allow to determine the parameter $\sin ^{2} \theta_{13}$ and to exclude the approach based on the evolution of the mixed neutrino states in time (see Section 2I). However, a negative result of such an experiment could be the consequence of the smallness of the parameter $\sin ^{2} \theta_{13}$. Thus, in the case of a negative result of the experiment definite conclusions on the fundamentals of neutrino oscillations can be drawn only if the parameter $\sin ^{2} \theta_{13}$ will be measured in future reactor (DOUBLE CHOOZ [28], Daya Bay [29]) or accelerator (T2K [30], Nova [31]) experiments.

2. We have considered an experiment, proposed in [16], on the search for neutrino oscillations driven by the 'large' $\Delta m_{23}^{2}$ and based on recoilless resonant absorption of $\bar{\nu}_{e}$. The baseline of this experiment is $\sim 10 \mathrm{~m}$. In such an experiment the effect of neutrino oscillations will be small (if present at all) because the amplitude of the oscillations is limited 
by the upper bound of the CHOOZ experiment $\left(\sin ^{2} 2 \theta_{13} \lesssim 2 \cdot 10^{-1}\right)$. The question arises if a similar oscillation experiment driven by the "small" $\Delta m_{12}^{2}$ could be performed. Such an experiment would require an about 30 times larger baseline, i.e., $\sim 300 \mathrm{~m}$. Because tritium acts as a point-like source the expected number of neutrino events in such an experiment will be $\sim 1000$ times smaller than in the 10 m-baseline experiment.

It was estimated in [16] that with a $10 \mathrm{~m}$ baseline about $10^{3} \bar{\nu}_{e^{-}}$ captures/day can be expected. Thus, in an experiment with a baseline of $\sim 300 \mathrm{~m}$ only about 1 capture/day could be observed. If we neglect the small contributions of the terms proportional to $\sin ^{2} \theta_{13}$ we find from eq.([6) for the $\bar{\nu}_{e}$ survival probability the following expression

$$
P\left(\bar{\nu}_{e} \rightarrow \bar{\nu}_{e}\right)=1-\frac{1}{2} \sin ^{2} 2 \theta_{12}\left(1-\cos \Delta m_{12}^{2} \frac{L}{2 E}\right) .
$$

Taking into account that the amplitude of the neutrino oscillations is large in this case (see eq.(2) ), such an experiment might still be feasible although we regard the estimate given in [16] as rather optimistic [17].

Let us note that according to eq.(27) the uncertainty of the energy of the antineutrinos emitted without recoil is much smaller than

$$
\frac{\Delta m_{12}^{2}}{2 E} \simeq 2.1 \cdot 10^{-9} \mathrm{eV}
$$

Thus, all our arguments given above for the possibilities to distinguish different approaches to the physics of neutrino oscillations are applicable also in this case. Notice also that two detectors of the same kind would allow to record the antineutrinos at two distances $(\sim 10 \mathrm{~m}$ and $\lesssim 300 \mathrm{~m})$.

3. We considered here different assumptions on the propagation of neutrino states (in time or in space and time). Different assumptions on the propagation of neutrino states give the same transition probabilities in the case of standard neutrino oscillation experiments. We have shown that a recently proposed Mössbauer-type neutrino experiment [16, 17] could allow to distinguish the different fundamental assumptions on the propagation of neutrinos with definite masses.

We thank T. Schwetz for fruitful discussions. S. Bilenky acknowledges the ILIAS program for the support and the TRIUMF Theory group for the hospitality. 


\section{References}

[1] Super-Kamiokande Collaboration, Y. Ashie et al., Phys. Rev. Lett. 93 (2004) 101801; Phys. Rev. D71 (2005) 11205.

[2] SNO Collaboration, Phys. Rev. Lett. 87 (2001) 071301; 89 (2002) 011301 ; 89 (2002) 011302; 92 (2004) 181301.

[3] KamLAND Collaboration, T.Araki et al., Phys. Rev. Lett. 94 (2005) 081801; hep-ex/0406035.

[4] B. T. Cleveland et al., Astrophys. J. 496 (1998) 505.

[5] GALLEX Collaboration, W. Hampel et al., Phys. Lett. B 447 (1999) 127 ; GNO Collaboration, M. Altmann et al., Phys. Lett. B 490 (2000) 16 ; Nucl. Phys. Proc. Suppl. 91 (2001) 44; Phys. Lett. B 616 (2005) 174.

[6] SAGE Collaboration, J. N. Abdurashitov et al., Phys. Rev. C 60 (1999) 055801; Nucl. Phys. B (Proc. Suppl.) 110 (2002) 315; Nucl. Phys. B (Proc. Suppl.) 118 (2003) 39.

[7] Super-Kamiokande Collaboration, S. Fukuda et al., Phys. Rev. Lett. 86 (2001) 5651.

[8] K2K Collaboration, M.H. Ahn et al., Phys. Rev. Lett. 90 (2003) 041801.

[9] MINOS Collaboration, D.G. Michael et al. arXiv:hep-ex/0607088.

[10] MiniBooNE Collaboration, A.A. Aguilar-Arevalo et al., arXiv: 0704.1500 v1 [hep-ex] 11 Apr 2007.

[11] LSND Collaboration, A. Aguilar et al., Phys. Rev. D 64 (2001) 112007; G. Drexlin, Nucl. Phys. B (Proc. Suppl.) 118 (2003) 146.

[12] CHOOZ Collaboration, M. Apollonio et al., Phys. Lett. B 466 (1999) 415; M. Apollonio et al., Eur. Phys. J. C 27 (2003) 331; hep-ex/0301017.

[13] B. Pontecorvo, J. Exptl. Theoret. Phys. 33 (1957) 549. [Sov. Phys. JETP 6 (1958) 429 ]; J. Exptl. Theoret. Phys. 34 (1958) 247 [Sov. Phys. JETP 7 (1958) 172 ]. 
[14] Z. Maki, M. Nakagawa, and S. Sakata, Prog. Theor. Phys. 28 (1962) 870.

[15] S.M. Bilenky, C. Giunti, and W. Grimus. Prog. Part. Nucl. Phys. 43 (1999) 1.

[16] R.S. Raghavan, hep-ph/0601079.

[17] W. Potzel, Phys. Scr. T127 (2006) 85.

[18] S.M. Bilenky, F. von Feilitzsch, and W. Potzel, J. Phys. G: Nucl. Part. Phys. 34 (2007) 987; hep-ph/0611285.

[19] S.M. Bilenky and C. Giunti, Int. J. Mod. Phys. A16 (2001) 3931; hep-ph/0102320.

[20] N.N. Bogolubov and D.V. Shirkov, Introduction to the theory of quantized fields (Interscience, New York, 1980).

[21] J.J. Sakurai, Modern Quantum Mechanics, (Addison Wesley Publishing Company, New York, 1994).

[22] S.M. Bilenky and M.D. Mateev, hep-ph/0604044.

[23] S.M. Bilenky and B. Pontecorvo, Phys. Rep. 41 (1978) 225.

[24] H.Lipkin, Phys. Lett. B579 (2004) 355.

[25] B. Kayser, hep-ph/0506165.

[26] C. Giunti, hep-ph/0409230.

[27] L. Stodolsky, Phys. Rev. D58 (1998) 036006.

[28] DOUBLE CHOOZ collaboration, S.A. Dazeley et al. Proceedings of the 7th International Workshop on Neutrino Factories and Superbeams (NuFact 05), Frascati, Italy, 21-26 Jun 2005; hep-ex/0510060

[29] Jun Cao, Nucl. Phys. Proc. Suppl. 156 (2006) 229.

[30] T2K Collaboration, Y. Hayato et al., Nucl. Phys. B (Proc. Suppl.) 143 (2005) 269.

[31] D.S. Ayres et al. NOvA collaboration, hep-ex/0503053 